\title{
Some Taxonomic Recommendations and a Proposal of Neotype Strains for Nineteen Species of Enterobacteriaceae
}

\section{ICSB Subcommittee on Taxonomy of Enterobacteriaceae}

\begin{abstract}
It is recommended by the ICSB Subcommittee on the Taxonomy of Enterobacteriaceae that the organism originally named Aerobacter liquefaciens by Grimes and Hennerty be regarded as a member of the genus Serratia rather than Enterobacter and that the organism originally named Pseudomonas proteamaculans by Paine and Stansfield be regarded as a member of the genus Serratia rather than Erwinia. In these positions, the correct names of these organisms are Serratia liquefaciens (Grimes and Hennerty) Bascomb et al. and Serratia proteamaculans (Paine and Stansfield) Grimont et al., respectively. The Subcommittee also proposes the following strains as neotypes for their respective species: Erwinia ananas NCPPB $1846 ; E$. cancerogena NCPPB 2176; E. carotovora NCPPB $312 ; E$. dissolvens NCPPB 1850; $E$. herbicola NCPPB 2971; $E$. milletiae NCPPB $2519 ; E$. paradisiaca NCPPB $2511 ; E$. stewartii NCPPB $2295 ; E$. tracheiphila NCPPB 2452; Escherichia blattae CDC 9005-74; Klebsiella oxytoca ATCC 13182; $K$. ozaenae ATCC 11296; $K$. pneumoniae ATCC 13883; $K$. rhinoscleromatis ATCC 13884; Proteus mirabilis ATCC 29906; Providencia rettgeri ATCC 29944; $P$. stuartii ATCC 29914; Shigella flexneri ATCC 29903; and $S$. sonnei ATCC 29930.
\end{abstract}

The members of the ICSB Subcommittee on Taxonomy of the Enterobacteriaceae (ISTE) made the following recommendations and proposals at, and subsequent to, the 12th International Congress for Microbiology held at $\mathrm{Mu}$ nich, Germany, in September 1978.

(i) The ISTE recommended that, on the basis of the biochemical and genetic evidence $(2,32)$, the organism originally named Aerobacter $l i$ quefaciens by Grimes and Hennerty be regarded as a member of the genus Serratia rather than of the genus Enterobacter. In this position, the correct name of the organism is Serratia liquefaciens (Grimes and Hennerty) Bascomb et al. (Syn.: Aerobacter liquefaciens Grimes and Hennerty; Enterobacter liquefaciens (Grimes and Hennerty) Ewing).

(ii) The ISTE also recommended that, on the same basis mentioned above (18), the organism originally named Pseudomonas proteamaculans by Paine and Stansfield be regarded as a member of the genus Serratia rather than of the genus Erwinia. In this position, the correct name of the organism is Serratia proteamaculans (Paine and Stansfield) Grimont et al. (Syn.: Pseudomonas proteamaculans Paine and Stansfield; Erwinia proteamaculans (Paine and Stansfield) Dye).

(iii) The ISTE here proposes as neotypes those strains listed in Table 1. To our knowledge, strains on which the original descriptions of these species were based are no longer extant. With the exception of the Erwinia strains, the characters of the proposed neotype strains are given in Table 2. The cell morphology of these strains conforms to that given in Bergey's Manual (12) for the Enterobacteriaceae; motile cells are peritrichous. The characteristics of the $E r$ winia strains are given by Young et al. (36). Cultures of the proposed neotype strains are available from the culture collections cited in Table 1.

\section{REPRINT REQUESTS}

Address reprint requests to: Don J. Brenner, Center for Disease Control, 1-B311, Atlanta, GA 30333.

\section{LITERATURE CITED}

1. Abel, R. 1893. Bakteriologische Studien über Ozaena simplex. Zentralbl. Bakteriol. Parasitenkd. Infektionskr. Hyg. Abt. 1 Orig. 13:161-173.

2. Bascomb, S., S. P. Lapage, W. R. Willcox, and M. A. Curtis. 1971. Numerical classification of the tribe Kleb sielleae. J. Gen. Microbiol. 66:279-295.

3. Bergey, D. H., F. C. Harrison, R. S. Breed, B. W. Hammer, and F. M. Huntoon. 1923. Bergey's manual of determinative bacteriology, 1st ed. The Williams \& Wilkins Co., Baltimore.

4. Bergey, D. H., F. C. Harrison, R. S. Breed, B. W. Hammer, and F. M. Huntoon. 1925. Bergey's manual of determinative bacteriology, 2nd ed. The Williams \& Wilkins Co., Baltimore.

5. Brenner, D. J., G. R. Fanning, C. V. Miklos, and A G. Steigerwalt. 1973. Polynucleotide sequence relatedness among Shigella species. Int. J. Syst. Bacteriol. 23:1-7.

6. Brenner, D. J., J. J. Farmer III, G. R. Fanning, A. G. Steigerwalt, P. Klykken, H. G. Wathen, F. W. Hickman, and W. H. Ewing. 1978. Deoxyribonucleic 
TABLE 1. Proposed neotype strains of 19 species of Enterobacteriaceae ${ }^{a}$

\begin{tabular}{|c|c|c|}
\hline Species & Proposed neotype strain & $\begin{array}{l}\text { Reference to } \\
\text { genetic and/or } \\
\text { biochemical } \\
\text { characters of } \\
\text { the strain }\end{array}$ \\
\hline Erwinia ananas Serrano 1928 (29) & NCPPB $1846=$ PDDCC 1850 & 36 \\
\hline E. cancerogena Urošefić 1966 (34) & NCPPB $2176=$ PDDCC 5706 & 36 \\
\hline $\begin{array}{l}\text { E. carotovora (Jones 1901) Bergey et al. } \\
1923(3,22)\end{array}$ & NCPPB $312=$ PDDCC $5702=$ ATCC 15713 & 36 \\
\hline $\begin{array}{l}\text { E. dissolvens (Rosen 1922) Burkholder } \\
1948(9,27)\end{array}$ & NCPPB $1850=$ PDDCC $1570=A T C C 23373$ & 32,36 \\
\hline $\begin{array}{l}\text { E. herbicola (Löhnis 1911) Dye } 1964 \text { (15, } \\
\text { 25) }\end{array}$ & NCPPB $2971=$ PDDCC 272 & 36 \\
\hline $\begin{array}{l}\text { E. milletiae (Kawakami and Yoshida } \\
\text { 1920) Magrou } 1937(23,26)\end{array}$ & NCPPB 2519 = PDDCC 5843 & 36 \\
\hline $\begin{array}{l}\text { E. paradisiaca Fernandez-Borrero and } \\
\text { Lopez-Doque } 1970(17)\end{array}$ & NCPPB 2511 & 36 \\
\hline $\begin{array}{l}\text { E. stewartii (Smith 1898) Dye } 1963 \text { (14, } \\
\text { 31) }\end{array}$ & NCPPB $2295=$ PDDCC $257=$ ATCC 8199 & 36 \\
\hline $\begin{array}{l}\text { E. tracheiphila (Smith 1895) Bergey et } \\
\text { al. } 1923(3,30)\end{array}$ & NCPPB $2452=$ PDDCC 5845 & 36 \\
\hline $\begin{array}{l}\text { Escherichia blattae Burgess et al. } 1973 \\
\text { (8) }\end{array}$ & CDC 9005-74 & 8 \\
\hline $\begin{array}{l}\text { Klebsiella oxytoca (Flügge 1886) Bren- } \\
\text { ner et al. } 1977(7)\end{array}$ & ATCC 13182 & 21 \\
\hline $\begin{array}{l}K . \text { ozaenae (Abel 1893) Bergey et al. } \\
1925(1,4)\end{array}$ & ATCC $11296=$ NCTC 5050 & \\
\hline $\begin{array}{l}\text { K. pneumoniae (Schroeter 1886) Trevi- } \\
\text { san } 1887(28,33)\end{array}$ & $\begin{array}{l}\text { ATCC } 13883=\text { NCTC } 9633=\text { DSM } 30104=\text { CDC } \\
298-53\end{array}$ & 13 \\
\hline K. rhinoscleromatis Trevisan 1887 (33) & ATCC $13884=$ NCTC 5046 & 13 \\
\hline Proteus mirabilis Hauser $1885(20)$ & ATCC 29906 & 7 \\
\hline $\begin{array}{l}\text { Providencia rettgeri (Hadley et al. 1918) } \\
\text { Brenner et al. } 1978(6,19)\end{array}$ & ATCC 29944 & 7 \\
\hline $\begin{array}{l}\text { P. stuartii (Buttiaux et al. 1954) Ewing } \\
1962(10,16)\end{array}$ & ATCC 29914 & 7 \\
\hline $\begin{array}{l}\text { Shigella flexneri Castellani and Chal- } \\
\text { mers } 1919 \text { (11) }\end{array}$ & ATCC 29903 & 5 \\
\hline $\begin{array}{l}\text { S. sonnei (Levine 1920) Weldin } 1927 \text { (24, } \\
\text { 35) }\end{array}$ & ATCC 29930 & 5 \\
\hline
\end{tabular}

a Abbreviations: ATCC, American Type Culture Collection, Rockville, Md.; CCM, Czechoslovak Collection of Microorganisms, 662 Brno, tr. Obrancu miru 10, Czechoslovakia; IIUP, Istituto di Igiene, Facoltà di Scienze, Università, Perugia, Italy; ISM, Istituto Sieroterapico Milanese "S. Belfanti," Milan, Italy; ISVT SCLAVO, Istituto Sieroterapico Vaccinogeno Toscano "SCLAVO," Siena, Italy; NCIB, National Collection of Industrial Bacteria, Aberdeen, Scotland; NCTC, National Collection of Type Cultures, Central Public Health Laboratory, London NW9 5HT, England.

acid relatedness of Proteus and Providencia species. Int. J. Syst. Bacteriol. 28:269-282.

7. Brenner, D. J., J. J. Farmer III, F. W. Hickman, M. A. Asbury, and A. G. Steigerwalt. 1977. Taxonomic and nomenclature changes in Enterobacteriaceae. CDC Publications, Atlanta, Ga.

8. Burgess, N. R. H., S. N. McDermott, and J. Whiting. 1973. Anaerobic bacteria occurring in the hind-gut of the cockroach, Blatta orientalis. J. Hyg. 71:1-7.

9. Burkholder, W. H. 1948. Genus I. Erwinia Winslow et al., p. 463-478. In R. S. Breed, E. G. D. Murray, and A. P. Hitchens (ed.), Bergey's manual of determinative bacteriology, 6 th ed. The Williams \& Wilkins Co., Baltimore.

10. Buttiaux, R., R. Osteux, R. Fresnoy, and J. Moriamez. 1954. Les propriétés biochimiques charactéristiques du genre Proteus. Inclusion souhaitable des Providencia dans celui-ci. Ann. Inst. Pasteur (Paris) $87: 375-386$.
11. Castellani, A., and A. J. Chalmers. 1919. Manual of tropical medicine, 3rd ed. Williams Wood and Co., New York.

12. Cowan S. T. 1974. FAMILY I. ENTEROBACTERIACEAE RAHN, 1937, 281 Nom. gen. cons. Opin. 15, Jud. Comm. 1958, 73, p. 290-340. In R. E. Buchanan and N. E. Gibbons (ed.), Bergey's manual of determinative bacteriology, 8th ed. The Williams \& Wilkins Co., Baltimore.

13. Cowan, S. T., K. J. Steel, C. Shaw, and J. P. Duguid. 1960. A classification of the Klebsiella group. J. Gen. Microbiol. 23:601-612.

14. Dye, D. W. 1963. The taxonomic position of Xanthomonas stewartii (Erw. Smith 1914) Dowson 1939. N. Z. J. Sci. 6:495-506.

15. Dye, D. W. 1964. The taxonomic position of Xanthomonas trifolii (Huss 1907) James 1955. N Z. J. Sci. 7: 261-269.

16. Ewing, W. H. 1962. The tribe Proteeae: its nomenclature 


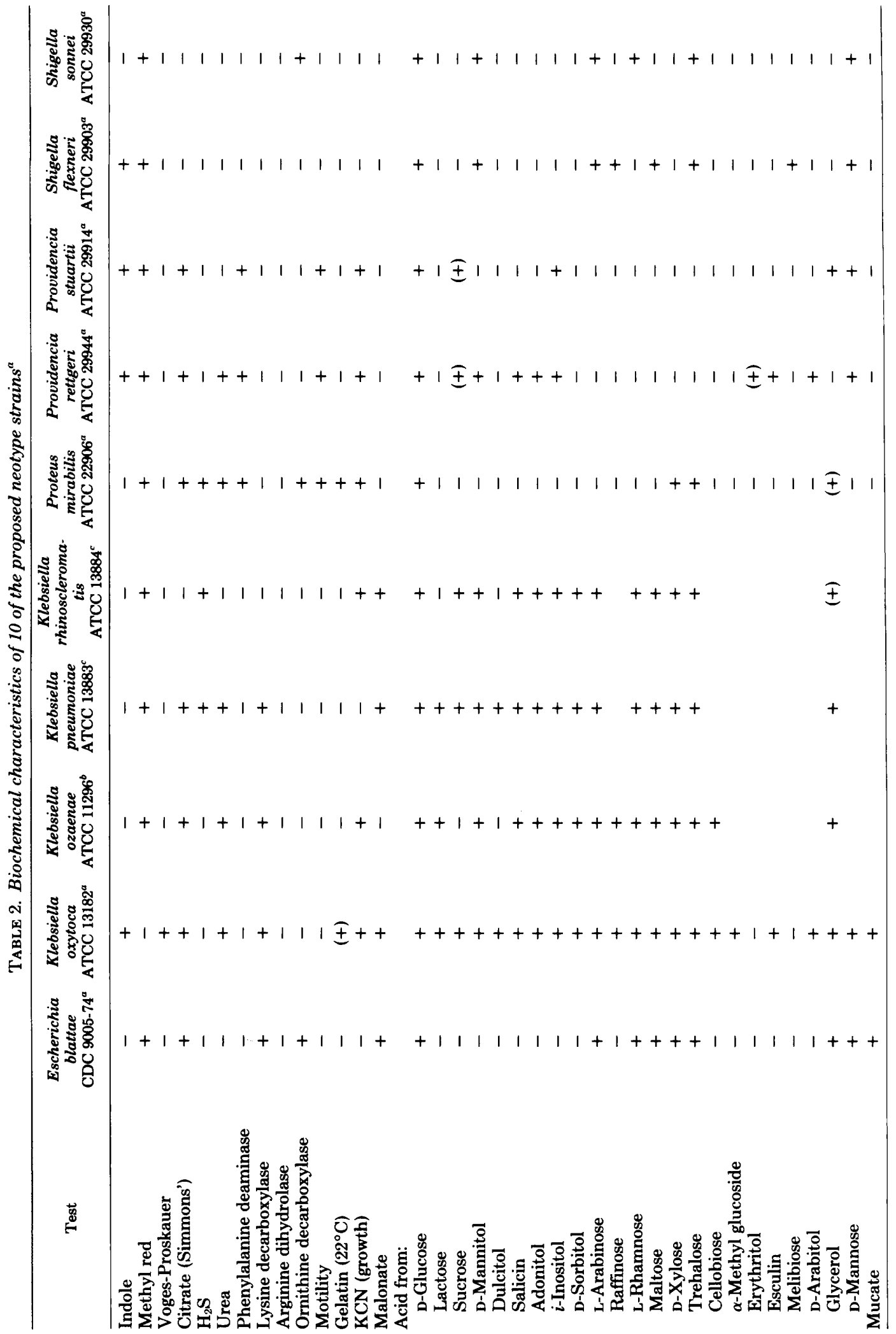




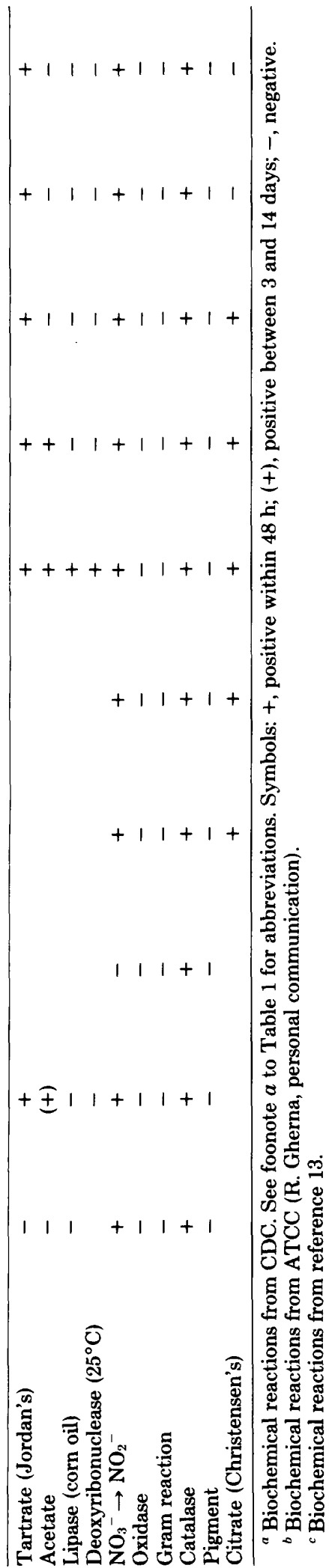

and taxonomy. Int. Bull. Bacteriol. Nomencl. Taxon. 12:93-102.

17. Fernandez-Borrero, O., and S. López-Duque. 1970 Pudrición acuosa de suëdo tallo del platano (Musa paradisiaca) causada por Erwinia paradisiaca n. sp. Cenicafe 21:3-14.

18. Grimont, P. A. D., F. Grimont, and M. P. Starr. 1978. Serratia proteamaculans (Paine and Stansfield) comb. nov., a senior subjective synonym of Serratia liquefa. ciens (Grimes and Hennerty) Bascomb et al. Int. J Syst. Bacteriol. 28:503-510.

19. Hadley, P, M. W. Elkins, and D. W. Caldwell. 1918 The colon-typhoid intermediates as causative agents of disease in birds. R. I. Agric. Exp. Stn. Bull. 174:1-216.

20. Hauser, G. 1885. Über Fäulnisbakterien und deren Beziehungen zur Septicämie. Ein Beitrag zur Morphologie der Spaltpilze. Vogel, Leipzig.

21. Jain, K., K. Redsak, and W. Mannheim. 1974. Differentiation of the Oxytocum group from Klebsiella by deoxyribonucleic acid-deoxyribonucleic acid hybridization. Int. J. Syst. Bacteriol. 24:402-407.

22. Jones, L. R. 1901. Bacillus caratovorus n. sp., die Ursache einer weichen Faulnis der Mohre. Zentralbl. Bakteriol. Parasitenkd. Infektionskr. Hyg. Abt. 2 7:1221 .

23. Kawakami, K., and S. Yoshida. 1920. Bacterial gall on Milletia plant (Bacillus milletiae n. sp.). Bot. Mag. (Tokyo) 34:110-115.

24. Levine, M. 1920. Dysentery and allied bacilli. J. Infect. Dis. 27:31-39.

25. Löhnis, F. 1911. Landwirtschaftlichbakteriologisches Praktium. Gebrüder Borntraeger, Berlin.

26. Magrou, J. 1937. In Hauduroy et al. (ed.), Dictionnaire des bactéries pathogènes. Masson and Co., Paris.

27. Rosen, H. R. 1922. The bacterial pathogen of corn stalk rot. Phytopathology 12:497-499.

28. Schroeter, J. 1886. In F. Cohn (ed.), Kryptogamen flora von Schlesien. Bd. 3, Heft 3, Pilze. J. U. Kern's Verlag, Breslau.

29. Serrano, F. B. 1928. Bacterial fruitlet brownrot of pineapple in the Philippines. Philipp. J. Sci. 36:271305.

30. Smith, E. F. 1895. Bacillus tracheiphilus sp. nov. die Ursache des Verwelkens verschiedener Curcurbitaceen. Zentralbl. Bakteriol. Parasitenkd. Infektionskr. Hyg. Abt. 2 7:364-373.

31. Smith, E. F. 1898. Notes on Stewart's sweet corn germ, Pseudomonas stewartin. sp. Proc. Am. Assoc. Adv. Sci. 47:422-426.

32. Steigerwalt, A. G., G. R. Fanning, M. A. Fife-Asbury, and D. J. Brenner. 1976. DNA relatedness among species of Enterobacter and Serratia. Can J. Microbiol. 22:121-137.

33. Trevisan, V. 1877. Sul micrococco della rabbia e sulla possibilità di riconoscere durante il periodo d'incubazione, dall'esame del sangue della persona morsicata, se ba contratta l'infezione rabbica. R. C. Inst. Lomb. (Ser. II) 20:797-804.

34. Urošefić, B. 1966. Canker of poplar caused by Erwinia cancerogena $n$. sp. Lesn. Cas. 12:493-505.

35. Weldin, J. C. 1927. The colon-typhoid group of bacteria and related forms. Relationships and classification. Iowa State J. Sci. 1:121-197.

36. Young, J. M., D. W. Dye, J. F. Bradbury, C. G. Panagopoulos, and C. F. Robbs. 1978. A proposed nomenclature and classification for plant pathogenic bacteria. N. Z. J. Agric. Res. 21:153-177.

Don J. Brenner for the ISTE 